\section{FoliaPhoniatrica et Logopaedica}

\section{Reiss}

Klinik und Poliklinik für

Hals-Nasen-Ohren-Heilkunde,

Universität Dresden, Deutschland

\title{
Aktuelle Untersuchungen zur Präferenzohrigkeit
}

\section{Key Words}

Auditory lateralization $\cdot$ Ear preference $\cdot$ Handedness

\section{Zusammenfassung}

Sensorische Asymmetrien zeigen sich vor allem im Bereich der Augen und der Ohren. Es gibt verschiedene Möglichkeiten, diese Asymmetrien zu untersuchen. Hierbei kann man Präferenz-, Leistungstests and sensorische Tests unterscheiden. Im Gegensatz zur Präferenz der Augen wurde die Ohrpräferenz bisher nur wenig berücksichtigt. Das Ziel dieser Studie ist es, einige neue Aspekte zur Untersuchung vorzulegen. Wir konnten in der Literatur insgesamt 13 verschiedene Tests zur Bestimmung der Präferenzohrigkeit nachweisen: «Schublade», «Tischdecke», «Schach-

\section{Current Aspects of Ear Preference}

Sensory asymmetries are most prominent with respect to the eyes and ears. There are various ways to investigate these asymmetries. The tests used for this purpose can be classified as preference, performance and sensory tests. Contrary to eyedness, earedness has received little attention. The objective of this study is to provide a few new investigatory aspects. We were able to identify 13 different tests for the determination of earedness in the literature: 'drawer', 'tablecloth', 'box', 'stop watch', 'telephone receiver', 'wall phone', 'earphone', tel», «Stoppuhr», «Telefonhörer», «Wandtelefon», «Ohrstecker», «Tür», «Herzschlag», «Taschenradio», «Stimme», «Wand» und «Kopfbewegung». Bei insgesamt 300 Studenten wurde nun die Brauchbarkeit dieser 13 Testproben geprüft. Es konnte eine hohe Beziehung zwischen «Stoppuhr», «Herzschlag», «Tür» und «Schachtel» nachgewiesen werden, nicht jedoch zwischen Telefonieren und Ohrigkeit. Es wird vorgeschlagen, die Ohrigkeit mit einer Testbatterie zu bestimmen, welche vier Testproben umfasst ( $«$ Stoppuhr», «Herzschlag», «Tür» und «Schachtel»).

'door', 'heartbeat', 'transistor radio', 'voice', 'wall' and 'head movement'. A study including 300 student test subjects was done to determine the usefulness of this battery of 13 tests. A high correlation was found between 'stop watch', 'heartbeat', 'door' and 'box', but not between telephoning and earedness. The determination of earedness by a battery comprised of 4 tests ('stop watch', 'heartbeat', 'door' and 'box') is suggested.

Dem Andenken an Prof. M.P. Bryden (1934-1996) gewidmet.

\begin{tabular}{ll}
\hline KARGER & (1 1998 S. Karger AG, Basel \\
Fax + 41 61 306 12 34 & 1021-7762/98/0501-0019\$15.00/0 \\
$\begin{array}{l}\text { E-Mail karger@karger.ch } \\
\text { www.karger.com }\end{array}$ & $\begin{array}{l}\text { This article is also accessible online at: } \\
\text { http://BioMedNet.com/karger }\end{array}$
\end{tabular}

Dr. med. M. Reiss

Klinik und Poliklinik für Hals-Nasen-Ohren-Heilkunde

Universitätsklinikum «Carl Gustav Carus» Dresden

Fetscherstrasse 74, D-01307 Dresden (Deutschland)

Tel. 03514584420 , Fax 03514584326 


\section{Etude actuelles sur la latéralité auditive}

Les asymétries sensorielles se manifestent surtout au niveau des yeux et des oreilles. Il existe plusieurs façons d'étudier des asymétries. On distingue les tests de latéralité, les tests de performance et les tests sensoriels. Contrairement à la latéralité visuelle, la latéralité auditive a été peu étudiée jusqu'ici. Cette étude a pour but de présenter des aspects nouveaux dans ce domaine. Nous avons retrouvé dans la littérature 13 tests au total pour la détermination de la latéralité auditive: «le tiroir», «la nappe», «la boîte», «le chronomètre», «l'écouteur téléphonique», «le téléphone mural»,

\section{Einleitung}

Die überwältigende Mehrheit der Menschen benutzt zum Schreiben und für andere koordinierte einhändige Tätigkeiten fast ausschliesslich die rechte Hand [1-3]. Aufgrund von kulturvergleichenden Studien wird angenommen, dass $90 \%$ der Menschen Rechtshänder sind [4-7]. Die Händigkeit ist aber nur ein besonders bekanntes Zeichen der sogenannten Hemisphärendominanz bzw. -spezialisierung. Daneben gibt es weitere funktionelle Asymmetrien, die man grob in motorische und sensorische einteilen kann [3, 812]. Die motorischen Asymmetrien umfassen neben der Händigkeit auch die Beinigkeit, die Asymmetrie des Gaumensegels und die Asymmetrie der Stimmbandbeweglichkeit [3]. $\mathrm{Zu}$ den sogenannten sensorischen Asymmetrien zählen insbesondere die Äugigkeit und die Ohrigkeit $[12,13]$. Bei der funktionellen Asymmetrie des Ohres bzw. Hörorgans unterscheidet man einmal die Präferenzohrigkeit, die Asymmetrie bei dem dichotischen Hörtest und die Asymmetrie der Hörschärfe, welche insbesondere im Hochtonbereich ab $8 \mathrm{kHz}$ zu beobachten ist $[1,8,9,14-16]$.

Unter Präferenzohrigkeit versteht man die bevorzugte Benutzung eines Ohres in Situationen, bei denen beide Ohren auch simultan «l'appareil auditif», «la porte», «les battements du cœur», «la radio portative», «la voix», «le mur», «le mouvement de la tête». L'utilité de ces 13 tests a été éprouvée chez 300 étudiants. Une relation significative avec la latéralité a été obtenue pour «le chronomètre», «les battements du cœur», «la porte» et «la bô̂te», mais pas pour le téléphone. Les auteurs proposent d'établir la latéralité auditive au moyen d'une batterie de 4 tests: «le chronomètre», «les battements du cœur», «la porte» et «la boîte».

verwendet werden können. Während der dichotische Hörtest einen breiten Raum in der neuropsychologischen Forschung einnimmt, wurde die Präferenzohrigkeit und die Asymmetrie der Hörschärfe nur relativ wenig untersucht $[1,2,12,17]$.

Die wohl umfangreichsten Untersuchungen zur Präferenzohrigkeit führten bisher Porac und Coren [2, 18], Coren [19] sowie Noonan und Axelrod [17] durch. So testeten Porac und Coren [2] 227 Probanden und konnten zwischen der Präferenzohrigkeit und der Asymmetrie beim dichotischen Hörtest eine gewisse Korrelation nachweisen: 73\% der Probanden zeigten einen Rechtsohrvorteil, 54\% waren rechtsohrig bezüglich der Präferenz und insgesamt $57 \%$ wiesen eine Übereinstimmung zwischen dichotischem Hörtest und Präferenzohrigkeit auf. Strauss [16], die die Präferenzhändigkeit, -beinigkeit, -äugigkeit und -ohrigkeit mit der Ohrdominanz auf der Basis des dichotischen Hörtests untersuchte, konnte zeigen, dass von den vier Präferenzasymmetrien die Ohrigkeit am besten mit dem Leistungstest korrelierte [7, 16].

Noonan und Axelrod [17] wiesen mit verschiedenen Tests nach, dass die Ohrpräferenz nur geringfügig durch Umwelteinflüsse beeinflusst wird. Die Autoren untersuchten 373 gesunde junge Erwachsene, wobei zur Bestim- 
Tabelle 1. Verschiedene Tests zur Bestimmung der Präferenzohrigkeit und wie häufig sie in der Literatur verwendet wurden

\begin{tabular}{|c|c|c|}
\hline Präferenztest & Abkürzung & $\begin{array}{l}\text { Häufigkeit in der } \\
\text { Literatur } \\
\text { (Anzahl der Studien) }\end{array}$ \\
\hline Nach einer Stoppuhr horchen & Stoppuhr & 8 \\
\hline $\begin{array}{l}\text { Richtung der Kopfbewegung, die d } \\
\text { Proband auf einen von hinten } \\
\text { kommenden Ton ausführt }\end{array}$ & Kopfbewegung & 6 \\
\hline $\begin{array}{l}\text { Horchen nach einer Uhr, die sich u } \\
\text { Tischdecke befindet }\end{array}$ & Tischdecke & 2 \\
\hline $\begin{array}{l}\text { Horchen nach einer Uhr, die sich in } \\
\text { Schublade befindet }\end{array}$ & Schublade & 1 \\
\hline $\begin{array}{l}\text { Horchen nach einer Uhr, die sich in } \\
\text { Schachtel befindet }\end{array}$ & Schachtel & 8 \\
\hline $\begin{array}{l}\text { Benutztes Ohr beim Telefonieren } \mathrm{n} \\
\text { Tischgerät (Handhörer) }\end{array}$ & Telefonhörer & 2 \\
\hline $\begin{array}{c}\text { Benutztes Ohr beim Telefonieren } n \\
\text { Wandtelefon (fixierter Hörer) }\end{array}$ & Wandtelefon & 2 \\
\hline Nach einem Herzschlag horchen & Herzschlag & 4 \\
\hline An einer Tür horchen & Tür & 8 \\
\hline Ohrstecker beim Radiohören & Ohrstecker & 7 \\
\hline An einer Wand horchen & Wand & 2 \\
\hline An einem Taschenradio horchen & Taschenradio & 1 \\
\hline Nach einer leisen Stimme horchen & Stimme & 1 \\
\hline
\end{tabular}
ziert].

Registriert wird jeweils das bevorzugte $\mathrm{Ohr}=$ Präferenz $(\mathrm{N}=27)$. Nach Reiss [nicht publi-

mung der Ohrpräferenz folgende Tests verwendet wurden: 1 . Horchen nach einer versteckten Stoppuhr auf einem Tisch, 2. die Verwendung eines Telefons, welches in der Mitte vor dem Proband angeordnet wurde und 3. das Horchen nach versteckten Lautsprechern. Noonan und Axelrod [17] zeigten, dass die Lateralität beim Telefonieren weniger durch die Händigkeit als durch das Schreiben und nicht durch die Ohrigkeit an sich beeinflusst wird.

Aktuelle Untersuchungen zur Präferenzohrigkeit
In der Literatur wurden die verschiedensten Testmethoden zur Bestimmung der Präferenzohrigkeit angeführt. Tabelle 1 zeigt eine Zusammenstellung der einzelnen in der Literatur angegebenen Testproben und wie oft sie von den einzelnen Autoren verwendet wurden. Weiterhin sind die einzelnen Abkürzungen, die im folgenden benutzt werden, angegeben. Es wird deutlich, dass die Proben «Stoppuhr», «Schachtel» und «Tür» am häufigsten verwendet wurden.

Folia Phoniatr Logop 1998:50:19-27 
Da im Gegensatz zu den Präferenzasymmetrien der Hände, der Augen und der Beine die Präferenzohrigkeit bisher relativ wenig beachtet wurde, ist das Ziel dieser Studie, weitere Untersuchungen zur Präferenzohrigkeit vorzulegen. Hierbei sollen die einzelnen Testproben näher betrachtet werden und die folgenden Fragen geklärt werden: Welche Testprobe ist für die Bestimmung der Präferenzohrigkeit am geeignetsten? Welche Proben besitzen eine geringe Wertigkeit? Kann die Probe «Telefonieren» zur Bestimmung der Präferenzohrigkeit benutzt werden?

\section{Methode}

\section{Probanden}

Es wurden insgesamt 300 Studenten (150 männliche und 150 weibliche), die an der Universität Halle Medizin studierten, untersucht. Das Alter betrug 2126 Jahre, das mittlere Alter 22,8 Jahre.

\section{Untersuchungsmethoden}

In einer grob orientierenden Hörprüfung ( 1 und 4 $\mathrm{kHz}$ ) wurde ausgeschlossen, dass eine Schwerhörigkeit vorliegt. Die entsprechende Testbatterie zur Bestimmung der Präferenzhändigkeit umfasste 9 Aufgaben: Zeichnen, Werfen, Zähneputzen, Nähen, Löffelhalten, Hämmern, Streichholzanzünden, Karten austeilen und einen Bleistiftstrich wegradieren [3, 20]. Die Probanden wurden augefordert, die einzelnen Proben dreimal auszuführen. Zeigte sich keine Seitenkonstanz, so wurden die Probanden ein viertes Mal getestet und dann der arithmetische Mittelwert gebildet. Linksauführung wurde jeweils mit einem Minuspunkt (-1), Rechtsausführung mit einem Pluspunkt (+1) und Beidseitigkeit bzw. keine Präferenz mit 0 bewertet. Die Punkte wurden entsprechend addiert und es resultierte eine Testskala, die von +9 bis -9 (extrem rechtshändig bis extrem linkshändig) reicht. Ein Proband ist rechtshändig, wenn eine Punktverteilung von +9 bis +4 vorliegt. Linkshändig ist ein Proband, wenn die Punktverteilung von -4 bis -9 beträgt. Die Probanden mit einem Punktwert von +3 bis -3 sind Beidhänder $[1,2$, 19, 20].

Neben der Präferenzhändigkeit wurde die Ohrbevorzugung von den in Tabelle 1 beschriebenen 13 Testproben ermittelt. Die Testung erfolgte hier wie bei der Präferenzhändigkeit. Berücksichtigt man alle 13
Testproben, so ergibt sich eine Lateralitätskala von +13 bis -13 (extrem rechtsohrig bis extrem linksohrig). Eine Rechtsohrigkeit liegt dann vor, wenn die Punktverteilung von +13 bis +5 reicht. Linksohrig ist der Proband, wenn die Punktverteilung von -6 bis -13 beträgt. Die Probanden mit einem Punktwert von +4 bis -5 sind beidohrig. Berücksichtigt man dagegen jede Testprobe einzeln, so resultiert jeweils ein Lateralitätsskala, die von -1 bis +1 reicht.

\section{Ergebnisse}

Berücksichtigt man alle 13 Testproben zur Bestimmung der Präferenzohrigkeit unter Verwendung einer symmetrischen Kategorienbildung [12], so sind insgesamt $62 \%(\mathrm{~N}=$ 186) der Probanden rechtsohrig, $21 \%(\mathrm{~N}=$ 63) linksohrig und 17\% (N=51) ohne Präferenz. Abbildung 1 zeigt die Verteilung der Ohrigkeit in graphischer Form, wobei der für eine Präferenzasymmetrie typische J-förmige Kurvenverlauf erkennbar ist [1, 2, 21]. Probanden mit extremer Seitigkeit sind relativ häufig, während diejenigen ohne oder mit geringerer Präferenz seltener sind. Da die Linksseiter gegenüber den Rechtsseitern in der Minderheit sind, resultiert eine J-förmige Kurve. Wäre die Anzahl der Rechts- und Linksseiter gleich, würde sich ein U-förmiger Kurvenverlauf ergeben [1,2].

Bezüglich der Händigkeit sind 84\% ( $\mathrm{N}=$ 252) der Probanden rechtshändig, $10 \%(\mathrm{~N}=$ 30) sind linkshändig und $6 \%(\mathrm{~N}=18)$ beidhändig. Tabelle 2 zeigt die Beziehung zwischen den einzelnen Ohrigkeitstestproben, der aus allen 13 Proben gebildeten Gesamtohrigkeit und der Händigkeit. Hierbei wurden Rechts-, Links- bzw. Beidseitigkeit mit $+1,-1$ bzw. 0 gleichgesetzt und die Korrelationskoeffizienten ( $r$ ) berechnet.

Zunächst fällt auf, dass fast alle Korrelationen ein positives Vorzeichen aufweisen. Ausserdem sind insgesamt 94 von den 105 möglichen Paarungen signifikant $(\mathrm{t}<0.05 ; \mathrm{N}=20)$ 


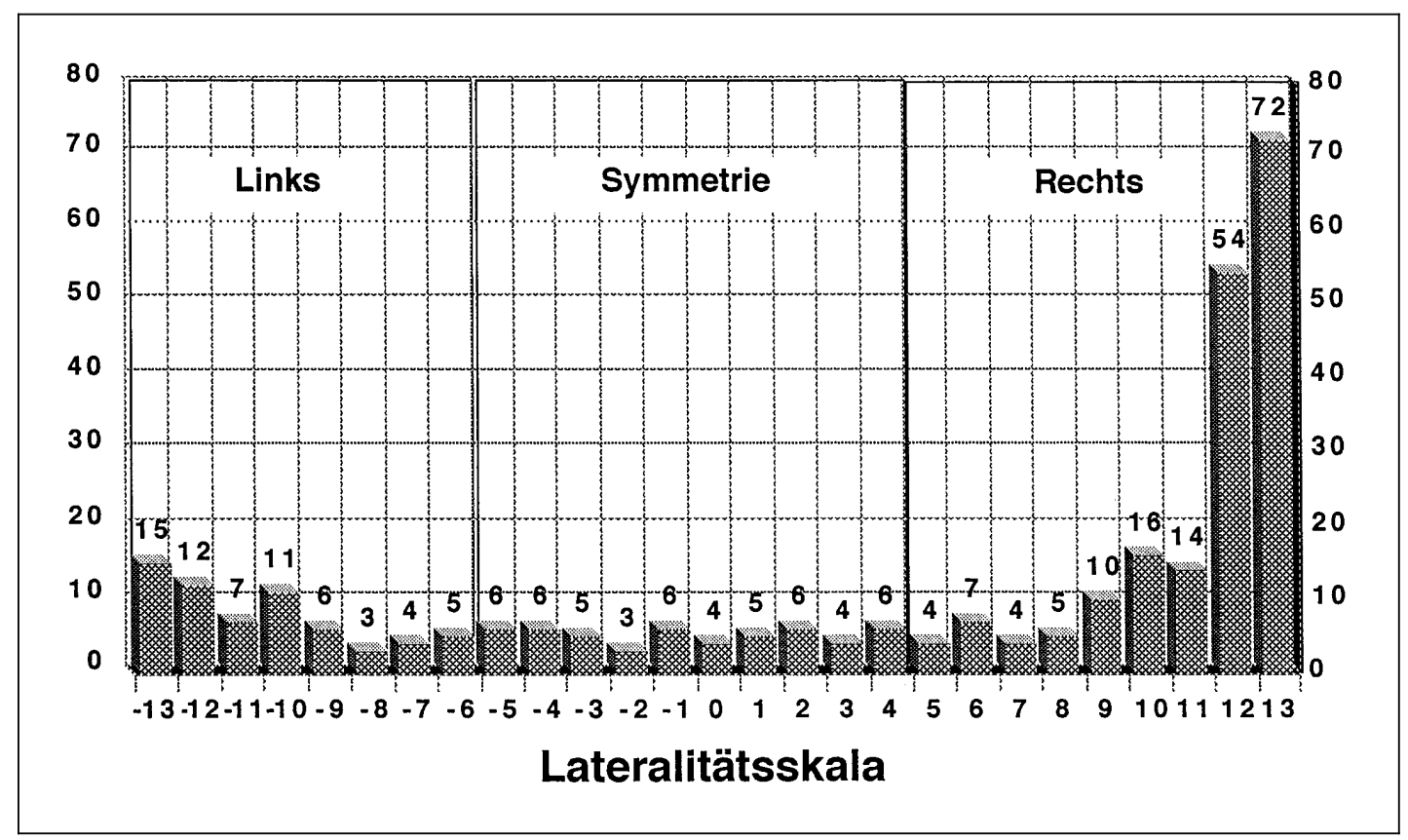

Abb. 1. Die Verteilung der Präferenzohrigkeit bestimmt auf der Basis von 13 Testproben bei 300 Studenten. Abszisse: Lateralitätsskala mit Unterteilung in Links-, Rechts- und Beidseitigkeit. Ordinate: Anzahl der jeweiligen Probanden (N).

bzw. hochsignifikant $(t<0,001 ; \mathrm{N}=74)$. Nur 2 Kombinationen weisen ein negatives Vorzeichen auf: «Händigkeit»-«Kopfbewegung» und «Händigkeit»-«Herzschlag». Hierbei besteht zwischen Händigkeit und Gesamtohrigkeit bzw. den 13 Ohrigkeitstestproben nur eine geringe, wenn auch z.T. signifikante Beziehung, wobei die Probe «Telefonhörer» am höchsten mit der Händigkeit $(r=0,210)$ korreliert.

Die Kombinationen «Schachtel»-«Tür» $(\mathrm{r}=0,821)$, «Tür»-«Herzschlag» $(\mathrm{r}=0,750)$, «Schachtel»-«Ohrstecker» $(\mathrm{r}=0,711)$, «Stoppuhr»-«Herzschlag» $(0,703)$, «Stoppuhr»-«Schachtel» $(\mathrm{r}=0,702)$ und «Schachtel»-«Herzschlag» $(r=0,677)$ weisen die höchsten Korrelationskoeffizienten auf.
Die Gesamtohrigkeit korreliert am höchsten mit den Proben «Stoppuhr» $(r=0,810)$, «Tür» $(\mathrm{r}=0,792)$, «Tischdecke» $(\mathrm{r}=0,690)$, «Herzschlag» $(r=0,652)$ und «Schachtel» $(r=$ 0,590). Dagegen sind die Korrelationskoeffizienten der Paarungen «Ohrigkeit»-«Telefonhörer» $(r=0,240)$, «Ohrigkeit»-«Kopfbewegung» $(r=0,290)$ und «Ohrigkeit»-«Stimme» $(r=0,371)$ am niedrigsten.

Auffällig ist, dass die Probe «Wandtelefon», im Gegensatz zur Probe «Telefonhörer», mit der Ohrigkeit mit $r=0,450$ relativ hoch korreliert. Die Probe Telefonhörer korreliert insbesondere mit den Proben «Wandtelefon» $(r=0,271)$, «Stimme» $(r=0,260)$ und «Taschenradio» $(r=0,156)$.

Die Proben «Kopfbewegung», «Stimme» und «Taschenradio» und «Tischdecke» kor- 
Tabelle 2. Korrelationsanalyse der einzelnen Ohrigkeitstests unter Berücksichtigung der Händigkeit $(\mathrm{N}=300)$

\begin{tabular}{|c|c|c|c|c|c|c|c|c|c|c|c|c|c|c|c|}
\hline & $\begin{array}{l}\text { Stopp- } \\
\text { uhr }\end{array}$ & $\begin{array}{l}\text { Kopfbe- } \\
\text { wegung }\end{array}$ & $\begin{array}{l}\text { Tisch- } \\
\text { decke }\end{array}$ & $\begin{array}{l}\text { Schub- } \\
\text { lade }\end{array}$ & $\begin{array}{l}\text { Schach- } \\
\text { tel }\end{array}$ & $\begin{array}{l}\text { Telefon- } \\
\text { hörer }\end{array}$ & $\begin{array}{l}\text { - Herz- } \\
\text { schlag }\end{array}$ & Tür & $\begin{array}{l}\text { Ohr- } \\
\text { stecker }\end{array}$ & Wand & Stimme & $\begin{array}{l}\text { Wand- } \\
\text { telefon }\end{array}$ & $\begin{array}{l}\text { Taschen } \\
\text { radio }\end{array}$ & $\begin{array}{l}\text { - Ohrig- } \\
\text { keit }\end{array}$ & $\begin{array}{l}\text { Händig- } \\
\text { keit }\end{array}$ \\
\hline $\begin{array}{l}\text { Stopp- } \\
\text { uhr }\end{array}$ & - & $0,322^{2}$ & $0,612^{2}$ & $0,560^{2}$ & $0,702^{2}$ & 0,098 & $0,703^{2}$ & $0,645^{2}$ & $0,556^{2}$ & $0,512^{2}$ & $0,301^{2}$ & $0,503^{2}$ & $0,456^{2}$ & $0,810^{2}$ & $0,156^{1}$ \\
\hline $\begin{array}{l}\text { Kopfbe- } \\
\text { wegung }\end{array}$ & & - & $0,310^{2}$ & $0,330^{2}$ & $0,430^{2}$ & $0,123^{1}$ & $0,290^{2}$ & 0,040 & $0,250^{2}$ & 0,010 & $0,264^{2}$ & $0,124^{1}$ & $0,211^{1}$ & $0,290^{2}$ & $-0,050$ \\
\hline $\begin{array}{l}\text { Tisch- } \\
\text { decke }\end{array}$ & & & - & $0,561^{2}$ & $0,160^{1}$ & $0,121^{1}$ & $0,190^{1}$ & $0,240^{2}$ & $0,300^{2}$ & $0,313^{2}$ & $0,432^{2}$ & $0,216^{2}$ & $0,297^{2}$ & $0,690^{2}$ & 0,002 \\
\hline $\begin{array}{l}\text { Schub- } \\
\text { lade }\end{array}$ & & & & - & $0,629^{2}$ & 0,101 & $0,522^{2}$ & $0,455^{2}$ & $0,365^{2}$ & $0,422^{2}$ & $0,391^{2}$ & $0,211^{2}$ & $0,344^{2}$ & $0,490^{2}$ & $0,195^{1}$ \\
\hline Schachte & & & & & - & $0,122^{1}$ & $0,677^{2}$ & $0,821^{2}$ & $0,711^{2}$ & $0,556^{2}$ & $0,362^{2}$ & $0,499^{2}$ & $0.270^{2}$ & $0,590^{2}$ & 0,112 \\
\hline $\begin{array}{l}\text { Telefon- } \\
\text { hörer }\end{array}$ & & & & & & - & 0,091 & $0,111^{1}$ & $0,190^{1}$ & $0,171^{1}$ & $0,260^{2}$ & $0,271^{2}$ & $0,156^{1}$ & $0,240^{2}$ & $0,210^{1}$ \\
\hline $\begin{array}{l}\text { Herz- } \\
\text { schlag }\end{array}$ & & & & & & & - & $0,750^{2}$ & $0,310^{2}$ & $0,411^{2}$ & $0,371^{2}$ & $0,420^{2}$ & $0,331^{2}$ & $0,652^{2}$ & $-0,020$ \\
\hline Tür & & & & & & & & - & $0,410^{2}$ & $0,671^{2}$ & $0,410^{2}$ & $0,306^{2}$ & $0,381^{2}$ & $0,792^{2}$ & 0,101 \\
\hline $\begin{array}{l}\text { Ohr- } \\
\text { stecker }\end{array}$ & & & & & & & & & - & $0,291^{2}$ & $0,290^{2}$ & $0,263^{2}$ & $0,412^{2}$ & $0,430^{2}$ & 0,090 \\
\hline Wand & & & & & & & & & & - & $0,322^{2}$ & $0,214^{2}$ & $0,395^{2}$ & $0,410^{2}$ & $0,126^{1}$ \\
\hline Stimme & & & & & & & & & & & - & $0,271^{2}$ & $0,344^{2}$ & $0,371^{2}$ & $0,132^{1}$ \\
\hline $\begin{array}{l}\text { Wand- } \\
\text { telefon }\end{array}$ & & & & & & & & & & & & - & $0,181^{1}$ & $0,450^{2}$ & $0,150^{1}$ \\
\hline $\begin{array}{l}\text { Taschen- } \\
\text { radio }\end{array}$ & & & & & & & & & & & & & - & $0,351^{2}$ & $0,127^{1}$ \\
\hline Ohrigkei & & & & & & & & & & & & & & - & $0,210^{1}$ \\
\hline Händigk & & & & & & & & & & & & & & & _ \\
\hline
\end{tabular}

relieren im Vergleich zu den Proben «Stoppuhr», «Schachtel», «Tür» oder «Herzschlag» insgesamt nur wenig mit den anderen Tests bzw. mit der Gesamtohrigkeit. Die Tests «Kopfbewegung» und «Tischdecke» zeigen ausserdem keine Beziehung zur Händigkeit.

Die Aufschlüsselung nach dem Geschlecht, auf die hier nicht näher eingegangen wird, ergibt keine statistisch signifikanten Unterschiede hinsichtlich der einzelnen geprüften Merkmale (Händigkeit, Ohrigkeit, einzelne Testproben bzw. Korrelationen). 


\section{Diskussion}

Die Präferenzohrigkeit ist als einfache und auch valide Methode ein möglicher Indikator zur Beurteilung der Hemisphärenspezialisierung bzw. -dominanz für Sprache. Sie ist einmal Bestandteil der Lateralität des Menschen insgesamt, andererseits ist sie eine mögliche Ergänzung zur Bestimmung der Hemisphärenspezialisierung mit dem dichotischen Hörtest $[1,2,8,9,12,16]$. Auch ist die Kenntnis der Präferenzohrigkeit bei der Indikationsstellung zur operativen Sanierung beidseitiger und symmetrischer Mittelohrerkrankungen nicht unerheblich [6]. Deshalb erscheint es wichtig, sowohl geeignete Proben zu identifizieren, als auch eine entsprechende Testbatterie zur Bestimmung der Präferenzohrigkeit vorzulegen. Während hinsichtlich der Händigkeit oder Äugigkeit einige Arbeiten über die Wertigkeit unterschiedlicher Testproben existieren $[1-3,18,19,21]$, gibt es bezüglich der Ohrigkeit fast keine Untersuchungen [2, $18,19]$. In dieser Studie werden erstmals alle verfügbaren bzw. bisher im Schrifttum verwendeten Testproben untersucht. Bisher wurde die Präferenzohrigkeit mit nur maximal drei Proben bestimmt [2, 19]. Einheitliche bzw. international anerkannte Richtlinien existieren weder für die Händigkeit noch für andere Präferenzasymmetrien [1, 3, 5, 10, 14 , 19, 20, 21].

Die hier angegebenen Daten für die Händigkeit bzw. die Präferenzohrigkeit stimmen im wesentlichen mit den in der Literatur ermittelten Verteilungen überein. Es wird deutlich, dass die Rechtsbevorzugung bei der Händigkeit viel höher ist als bei der Ohrigkeit $[1,4,10,14,16,18,19,22]$.

Ähnlich wie bei der Händigkeit kann man erkennen, dass die Grenzziehung zwischen den einzelnen Kategorien «rechts», «Symmetrie» und «links» problematisch ist und oftmals willkürlich gewählt werden muss. Am zweckmässigsten hat sich hierbei die sogenannte symmetrische Kategorienbildung bewährt, da hierbei allen drei Gruppen eine gleich breite Wertungsstrecke eingeräumt wird. Die ermittelten Werte für «rechts», «Symmetrie» bzw. «links» variieren dementsprechend mit der Grenzziehung [23].

Ähnlich wie bei der Händigkeit bzw. anderen asymmetrischen Merkmalen oder Testproben wurden relativ hohe Korrelationen ermittelt $[2,4,18]$. Allerdings zeigen sich bei genauerer Betrachtung erhebliche Unterschiede. Die Proben «Schachtel», «Tür», «Herzschlag»; «Ohrstecker» und «Stoppuhr» korrelieren am höchsten sowohl miteinander als auch mit der Gesamtohrigkeit, so dass sie als die Tests mit der höchsten «Wertigkeit» angesehen werden können. Damit sind sie zur Bestimmung der Präferenzohrigkeit besonders geeignet. Die Proben «Kopfbewegung», «Stimme», «Taschenradio» und «Tischdekke» sind dagegen nicht so brauchbar [17, 19].

Die hier demonstrierte geringe Korrelation zwischen Händigkeit und Ohrigkeit stimmt mit den Ergebnissen in der Literatur überein [1, 16, 19, 22]. Die Probe «Telefonhörer» weist die höchste Beziehung zur Händigkeit auf. Das kann als Indiz dafür gewertet werden, dass die Lateralität beim Telefonieren insbesondere durch die Händigkeit beeinflusst wird. Es wird dagegen deutlich, dass insbesondere die Proben, die relativ selten durch die Probanden angewendet werden bzw. die nicht «alltäglich» sind und die keine soziokulturelle Beeinflussung erfahren, nur wenig mit der Probe «Telefonhörer» korrelieren. Bei diesen Tests ist der Gebrauch der Hände ausgeschlossen. Die Probe «Telefonhörer» zeigt nur eine verhältnismässig geringe Korrelation zur Präferenzohrigkeit. Dagegen ist verwunderlich, dass das ebenso bei der Probe «Wandtelefon» der Fall ist, was möglicherweise auch durch die Beeinflussung des Telefonohres zurückzuführen ist. Andererseits 
zeigen verschiedene Proben eine gewisse Ähnlichkeit, wie z.B. «Stimme» und «Kopfbewegung», so dass die unterschiedlich hohen Korrelationskoeffizienten eigentlich nicht auftreten dürften. Möglicherweise spielen hier untersuchungstechnische Aspekte eine Rolle [2, 17]. Es sollte noch bemerkt werden, dass die Probe «Ohrstecker» in einer Zeit der binauralen Kopfhörerbenutzung nicht mehr zeitgemäss ist und deshalb nicht mehr verwendet werden sollte. Da diese Probe in der älteren Literatur relativ häufig benutzt wurde (Tab. 1), wurde sie in die Testbatterie aufgenommen. Erwähnenswert ist, dass sie mit zu den Proben mit der höchsten «Wertigkeit» zählt.

Auf der Grundlage der Untersuchungsergebnisse wird vorgeschlagen, die Präferenzohrigkeit mit einer Testbatterie zu bestimmen, die die Proben «Stoppuhr», «Schachtel», «Tür» und «Herzschlag» umfassen sollte. Hierdurch wird die Verwendung der Hände ausgeschlossen, so dass sie durch die Händigkeit nicht beeinflusst werden kann. Gleichzeitig handelt es sich um Proben, die zum Teil nicht alltagsüblich sind, so dass ein gewisser
Trainingseffekt wegfällt. Bestimmt man nun die Präferenzohrigkeit mit diesen vier Proben, so sind 63\% ( $\mathrm{N}=189)$ der Probanden rechtsohrig (Lateralitätsskala: +4 bis +2 ), $21 \%(\mathrm{~N}=63)$ linksohrig $(-4$ bis -2$)$ und $16 \%$ $(\mathrm{N}=48)$ beidohrig $(+1$ bis -1$)$, wobei ebenfalls eine symmetrische Kategorienbildung benutzt wurde. Diese Verteilung stimmt mit derjenigen überein, die unter Benutzung der 13 Testproben ermittelt wurde. Dementsprechend sind die Unterschiede nicht signifikant $\left(\chi^{2}=0,115 ; \mathrm{FG}=2, \mathrm{NS}\right)$.

Das Telefonieren, d.h. die Probe «Telefonhörer», ist aufgrund der möglichen Beeinflussung durch die Händigkeit nicht geeignet für die Bestimmung der Ohrigkeit. Idealerweise sollten natürlich ebenso viele Testproben wie zur Bestimmung der Händigkeit verwendet werden. Jedoch dürfte eine Bestimmung mit vier Tests («Stoppuhr», «Schachtel», «Tür» oder «Herzschlag») aufgrund der relativ hohen Korrelation ausreichend sein. Eine entsprechende Testbatterie zur Bestimmung der Gesamtlateralität, d.h. der Hand-, Bein-, Augen- und Ohrpräferenz, wäre dadurch auch nicht zu umfangreich [2, 8, 14, 19, 21].

\section{Literatur}

1 Bryden MP: Laterality: Functional Asymmetry in the Intact Brain. New York, Academic Press, 1992.

2 Porac C, Coren S: Lateral Preferences and Human Behavior. Berlin, Springer, 1981.

3 Reiss M: Untersuchungstechnische Aspekte motorischer Asymmetrien. Wien Med Wochenschr 1997;147: 275-278.

4 Bourassa DC, McManus IC, Bryden MP: Handedness and eye-dominance: A meta-analysis of their relationship. Laterality 1996;1:5-34.
5 Brito GN, Brito LS, Paumgartten FJ, Lins MF: Lateral preferences in Brazilian adults: An analysis with the Edinburgh Inventory. Cortex 1989;25:403-415.

6 Reiss M: Die praktische Bedeutung der Lateralität. Ärztl Jugendkd 1991;82:69-76.

7 Strauss E, Goldsmith SM: Lateral preferences and performance on non-verbal laterality tests in a normal population. Cortex 1987;23: 495-503.

8 Böhme G: Hochtonaudiometrie. I. Ohrigkeit und Lateralität. HNO 1978;26:312-315.
9 Bryden MP: Correlates of the dichotic right-ear effect. Cortex 1988; 24:313-319.

10 Groden G: Lateral preferences in normal children. Percept Mot Skills 1969;28:213-214.

11 Mandal MK, Pandey G, Singh SK, Asthana HS: Degree of asymmetry in lateral preferences: Eye, foot, ear. J Psychol 1992;126:155-162.

12 Reiss M: Untersuchungstechnische Möglichkeiten sensorischer Asymmetrien. Psycho 1996;22:584-588. 
13 Reiss M, Reiss G: Ocular dominance: Some family data. Laterality 1997;2:7-15.

14 Kovác D, Horkovic G: How to measure lateral preference. Stud Psychol 1970;12:5-11, 169-170.

15 Reiss M: Die Untersuchung der Hemisphärenspezialisierung beim Menschen. Z Ärztl Fortbild 1992; 86:157-162.

16 Strauss E: Hand, foot, eye and ear preferences and performance on a dichotic listening test. Cortex 1986; 22:475-482.
17 Noonan M, Axelrod S: Earedness (ear choice in monaural tasks): Its measurement and relationship to other lateral preferences. J Audit Res 1981;21:263-277.

18 Porac C, Coren S: Individual and familial patterns in four dimensions of lateral preference. Neuropsychologia 1979; 17:543-548.

19 Coren S: The lateral preference inventory for measurement of handedness, footedness, eyedness, and earedness: Norms for young adults. Bull Psychonomic Soc 1993;31:1-3.

20 Reiss M: Laterality of writing hand in East German samples. Percept Mot Skills 1997;84:474.
21 Chapman LJ, Chapman JP: The measurement of handedness. Brain Cogn 1987;6:175-183.

22 Gudmundsson E: Lateral preference of preschool and primary school children. Percept Mot Skills 1993; 77:819-828.

23 Oeser R: Händigkeitsstufen, Lebensalter und Geschlecht: Untersuchungen an 7- bis 17-jährigen Probanden. Ärztl Jugendkd 1973;64: 437-447.

\section{Ankündigung}

\section{Annelie-Frohn-Preis 1998}

Von der Deutschen Gesellschaft für Phoniatrie und Pädaudiologie und der Annelie-Frohn-Stiftung wird für das Jahr 1998 der Annelie-FrohnPreis ausgeschrieben. Die Dotation beträgt DM 10.000,-. Der Preis wird für eine herausragende wissenschaftliche Arbeit zur Thematik «Förderung hör- und/oder sprachgestörter Kinder» vergeben.

Bewerbungen sind an den Präsidenten der Deutschen Gesellschaft für Phoniatrie und Pädaudiologie, Herrn Prof. M. Gross, Fabeckstrasse 62, D-14195 Berlin (Deutschland), bis zum 30. April 1998 einzureichen. 